\title{
Ontología y Lenguaje de la Realidad Social
}

\author{
Ontology and LANGuage of Social Reality
}

Mg. Jorge Posada-Ramírez (gposada@uniquindio.edu.co) Programa de Filosofía, Facultad de Ciencias Humanas y de Bellas Artes, Universidad del Quindío (Armenia, Colombia)

\begin{abstract}
This paper shows, from the ontology and the philosophy of language, a series of characteristics of social sciences that proves the conceptual impossibility to join them with natural sciences as a unique science. Philosophical characteristics of social sciences' subjects (ontology of social reality), such as some features of the language that defines the social reality, illustrate that the structure of conceptual scheme of social sciences is, largely, incommensurable with the structure of natural sciences. So the text tries to explain, especially from the philosophical theses of John Searle, that the ontology of social sciences and the linguistic schemes in which is described and through which becomes the social reality, exhibit features that make about it unapproachable by natural sciences. The final objective around the over-documented methodological distinction between social and natural sciences, is to show reasons from ontology and philosophy of language for arguing the conceptual impossibility, but not methodological, for comparing both kinds of knowledge.
\end{abstract}

Keywords: social sciences, natural sciences, subjective ontology, language, John Searle.

\section{Resumen}

El siguiente texto muestra desde la ontología y la filosofía del lenguaje una serie de características de las ciencias sociales que al contrastarse con las ciencias naturales prueban la imposibilidad conceptual de conglomerarlas en una única ciencia. Las características filosóficas de los objetos de estudio de las ciencias sociales (ontología de la realidad social), así como ciertos rasgos del lenguaje que definen la realidad social, ilustra que la estructura del esquema conceptual de las ciencias sociales es, en buena medida, inconmensurable con el de las ciencias naturales. Así, el texto intenta mostrar, en especial desde propuesta del filósofo John Searle, que la ontología de las ciencias sociales, así como los esquemas lingüísticos en los que se describe y deviene la realidad social, exhiben rasgos que la hacen inabordable por las ciencias naturales. Lo que se espera aportar en torno a la muy documentada distinción metodológica entras ciencias sociales y ciencias naturales, son razones de naturaleza ontológica y desde la filosofía del lenguaje que argumentan la imposibilidad ya no metodológica, sino conceptual, de cotejar estos dos tipos de saber.

Palabras clave: ciencias sociales, ciencias naturales, ontología subjetiva, lenguaje, John Searle. 


\section{Introducción}

Guerras, conflictos, propiedad privada, bienes públicos, derechos, deberes, economía, dinero, matrimonio, desempleo, empresas, religiones, parlamentos, impuestos, divorcios, familia son unos escasos ejemplos de cosas que las personas hacen. Planetas, estrellas, átomos, movimiento de las placas tectónicas, océanos, electrones, protones son cosas que la gente no hace, sino que están dadas en la naturaleza. Aristóteles en la Ética a Nicómaco distinguió entre los sucesos que se dan en el mundo, originados por cierta dinamis en la materia que la fuerza a que se comporte de cierto modo, y los acontecimientos que ocurren en tanto el hombre es su principio causal: "como los hijos son de sus padres" (Aristóteles 1967:1201). Por ejemplo, la transformación de algunos fósiles en petróleo se debe a condiciones causales que determinan que algas y zooplancton se conviertan en hidrocarburos; diríamos, en términos de Aristóteles, a su dinamis; el cambio de un hombre de estar soltero a casado se debe a condiciones de abierta elección, que permiten decidir a una persona causar su nuevo estado civil. Como lo vio Aristóteles, la diferencia entre las cosas que pasan naturalmente y las que el hombre hace se debe a su historia causal. Las que tienen como origen causal regularidades naturales son sucesos, las que tienen como origen causal las elecciones y decisiones humanas son acciones. Así, la rotación de la luna es un suceso, las guerras son acciones. El filósofo de la acción Harry Frankfurt, siguiendo a Aristóteles, llama teorías causales a la posición filosófica que define la clase de eventos que en tanto causados por las creencias y deseos de las personas son considerados como acciones.

Frankfurt afirma: "El problema de la acción reside en explicar el contraste entre lo que hace un agente y lo que meramente le sucede, o entre los movimientos corporales que realiza y aquellos que se producen sin que él los provoque. De acuerdo con las teorías causales sobre la naturaleza de la acción -en la actualidad el enfoque más generalizado en la comprensión de este contraste-, la diferencia esencial entre los acontecimientos de ambos tipos se encuentra en sus historias causales previas: un movimiento corporal es una acción si y sólo si es el resultado de antecedentes de cierto tipo" (Frankfurt 2006:105).

Es recurrente que el enfoque causal asuma los antecedentes causales de las acciones como creencias y deseos, los que en conjunción producen el movimiento corporal de un agente y con ello una acción. Desde esta primera perspectiva filosófica puede decirse que las ciencias naturales describen y explican sucesos y las ciencias sociales describen y explican acciones. Las distinciones gruesas entre acciones y sucesos marcan diferencias entre las ciencias sociales y ciencias naturales. La diferencia más básica es la que nos ha sugerido Aristóteles: mientras las causas de los sucesos son regularidades naturales, las causas de las acciones son las decisiones humanas, decisiones que son originadas por las creencias y los deseos de las personas. Así, en tanto la fosilización en la tierra de algas y plancton se produce con independencia de los deseos y las creencias de las personas, para que se den el matrimonio o la guerra es necesaria la existencia de personas que alberguen y actúen por creencias y deseos. Creencias y deseos son las causas de las acciones, dos tipos de estados mentales que determinan la aparición de acciones, y que no son relevantes en la constitución de lo que podemos llamar sucesos.

Desde la idea de que las acciones son aquel tipo de eventos causados por las creencias y deseos de las personas, y que constituyen, grosso modo, el objeto de estudio de las ciencias sociales, y que los sucesos son aquel tipo de eventos en donde creencias y deseos son irrelevantes, se argumentan algunas diferencias filosóficas entre las ciencias sociales y las ciencias naturales. 


\section{Distinciones ontológicas}

La ontología es la rama de la filosofía que se dedica a reflexionar sobre los modos esenciales de existencia de las cosas. Por ejemplo, le es esencial a un triángulo para su existencia el estar conformado por tres ángulos. Su identidad depende de esta característica, que es tal, que sin ella simplemente no podría existir. Sólo existen los triángulos en tanto existan tres ángulos que delimitan entre sí un área. Es contingente para la existencia ontológica de lo que es triángulo si está hecho de madera, de bronce, de oro o si es un área del océano Atlántico delimitada por tres islas, pues no es un asunto de la ontología reparar en estas características específicas de los triángulos. La ontología busca identificar y aclarar las condiciones esenciales que determinan la identidad y la existencia de las cosas. Como reza en los libros de texto, la ontología es la rama de la filosofía que estudia lo que hace que las cosas sean lo que son. Para ser un triángulo no se necesita ser ni de madera, ni de bronce, ni delimitar un área del océano Atlántico, pero sí estar conformado por tres ángulos; este es uno de sus rasgos esenciales. Así, ontológicamente hablando, y esto es, señalando las condiciones esenciales de existencia, algo es un triángulo si y solo si es una figura de tres ángulos.

En filosofía las preguntas: qué es ser persona, qué es un estado mental, qué es ser un zombi, son preguntas ontológicas, y las respuestas que a ellas se dan, buscan establecer las condiciones esenciales que hacen que algo sea persona, un estado mental o un zombi. La pregunta ontológica que ahora se hace relevante es: ¿qué es ser un hecho de estudio para las ciencias sociales?

Los hechos que son objeto de estudio de las ciencias sociales igualmente deben revelar un modo de existencia esencial. El filósofo del lenguaje y de la mente John Searle, en su libro La construcción de la realidad social (1997), afirma que un rasgo esencial de los hechos sociales es la subjetividad, en sus palabras, "ontología subjetiva". Una condición esencial de existencia de las entidades que son objeto de estudio de las ciencias sociales es la existencia de los sujetos y su capacidad mental de causar acciones. Las ciencias sociales tratan sobre hechos que son causados por los sujetos, este es uno de los rasgos más relevantes de su existencia. Se hace más claro este rasgo cuando se contrasta con el campo de la realidad que es teorizada por las ciencias naturales, campo en el que la subjetividad de las personas, sus acuerdos, creencias y deseos, en tanto causas de acciones, no es relevante.

Desde Searle pude argüirse que lo ontológicamente objetivo es aquello que existe independientemente del acuerdo de los humanos. Él mismo propone la siguiente clasificación ontológica, la que presenta con el interés de explicar la región de la realidad que es creada por los intereses y creencias de las personas. Señala que hay hechos brutos y hechos institucionales. Los hechos brutos son aquellos que existen con independencia de los acuerdos y las convenciones humanas. Son hechos brutos el Monte Everest, el petróleo que yace bajo el suelo venezolano, el movimiento de las placas tectónicas, la resistencia de los materiales. Los hechos institucionales son aquellos que dependen de los acuerdos humanos. Las guerras, la propiedad privada, los Estados, las universidades, las teorías científicas, el lenguaje mismo, son hechos institucionales. Los hechos brutos son ontológicamente objetivos, en el sentido de que su modo de existencia no depende de las personas. Los hechos institucionales son ontológicamente subjetivos, en el sentido de que una condición esencial de su ser es la existencia de las personas. Existan o no existan personas, el punto más alto de la tierra o los fósiles existen, pero para que haya Estados, guerras y matrimonios es condición esencial la existencia de las personas. 
Tenemos la primera distinción filosófica entre las ciencias sociales y las ciencias naturales. Los objetos de estudio de las ciencias sociales son ontológicamente subjetivos, los objetos de estudio de las ciencias naturales son ontológicamente objetivos. Se podría pensar que la anatomía y la histología humana en tanto estudian objetos que dependen para su existencia de sujetos humanos, son ontológicamente subjetivas, y en consecuencia, están más cercanas a las ciencias sociales que naturales. No obstante, hay que acentuar que lo ontológicamente subjetivo son aquellas cosas que para su existencia dependen de los sujetos con sus creencias y deseos. Los seres humanos con sus tejidos no existen por acuerdos o convenciones humanas. Por esto, en tanto animales mamíferos, de cierta estructura anatómica y con un sistema nervioso central altamente desarrollado, los humanos somos ontológicamente objetivos. La misma capacidad mental que posibilita la existencia de las creencias y los deseos es ontológicamente objetiva, pues no es producto de un acuerdo o convención de las personas, sino de la evolución natural.

\section{Dispositivos mentales en la construcción de la ontología de las ciencias sociales}

La tesis gruesa que en este apartado se quiere mostrar es que las ciencias sociales estudian hechos que en esencia no son físicos, sino mentales; en contraste con las ciencias naturales, que estudian acontecimientos en esencia materiales, y no mentales. Se partirá de la explicación de John Searle de los hechos institucionales, que aquí junto con las acciones, presentamos como objeto de estudio de las ciencias sociales. A partir de los conceptos de: Intencionalidad, Reglas Constitutivas y Funciones de Estatus, se apoyará la tesis de que es condición necesaria para la existencia de los hechos sociales, ciertos dispositivos mentales que crean y sostienen la realidad social.

Parte de la ontología subjetiva (la que se revela en el matrimonio, en el dinero, los Estados), se constituye gracias a tres capacidades humanas, que dirigidas a los hechos brutos les cambia su configuración natural, tornándolos objetos institucionales, hechos de estudio de las ciencias sociales: Intencionalidad, Reglas Constitutivas y Funciones de Estatus. Para Searle, estas tres capacidades, por ejemplo, dirigidas al papel, transfiguran esas finas fibras de vegetal, en dinero, un objeto del mundo que si bien está hecho de papel, en su esencia ontológica no es papel. El papel no es dinero, ni el dinero es papel. Las ciencias naturales estudian objetos como las fibras vegetales, la pulpa de celulosa, la madera: ontología objetiva, y las ciencias sociales estudian objetos que como el dinero en esencia no es físico ni material: ontología subjetiva. Ahora bien, describir estas tres capacidades humanas que permiten la constitución de los hechos institucionales favorece la distinción entre ellos con respecto a lo que es estudiado por las ciencias naturales.

Intencionalidad. Es la capacidad humana, y seguramente de sistemas nerviosos centrales similares al de los humanos, de formar estados mentales que representan, se dirigen y versan sobre el mundo. Las personas, a diferencia de las rocas, los ríos y la gran mayoría de las restantes especies animales, tenemos la capacidad de representarnos, a través de estados mentales, el mundo. Así, podemos representarnos la Iluvia del 31 de octubre de 2020, nuestro deseo de que la selección colombiana de fútbol en el 2022 gane el Mundial. Estas cosas que aún no han pasado, a pesar de todo, pueden ser representadas por nosotros en estados mentales como el creer o desear. Creo que "va a llover el 31 de octubre de 2020", es un estado mental que representa un estado posible de cosas en el mundo, lo mismo el deseo de que "La selección colombiana de fútbol gane el Mundial de 2022". La Intencionalidad es justamente esa capacidad de nuestro cerebro de representar estados de cosas y posibles estados de cosas del mundo. 
Como afirma John Searle (1992), dado que los estados mentales intencionales tienen la capacidad de dirigirse a estados de cosas del mundo, puede representarlos y relacionarse con ellos a partir de este rasgo de representación. Así, estados mentales como el creer, el desear, son Intencionales; su rasgo característico consiste en estar en la mente como formas mentales que representan o versan sobre el mundo. Por esto, no hay creencias ni deseos a secas. Las creencias y los deseos siempre versan sobre algo o representan algo. Creo que: "va a llover el 31 de octubre de 2020", deseo que: "La selección colombiana de fútbol gane el mundial 2022", la lluvia de ese día señalado, ese equipo de fútbol, conformado por esos hombres de carne y hueso, son hechos físicos, sólo que en la creencia y en el deseo están representados mentalmente. Así, los estados mentales Intencionales son estados que representan y versan sobre cosas distintas de ellos mismos. Una cosa es el hecho físico de la lluvia y otro la creencia de que va a llover.

Reglas constitutivas. A través del lenguaje las personas pueden crear reglas que, en tanto se sigan, crean acciones no preexistentes antes de las reglas. La capacidad de formar reglas constitutivas es la que permite la creación de acciones como hacer goles, jugar ajedrez o nombrar presidentes. Antes de la constitución de las normas que definen qué es hacer un gol, qué es jugar ajedrez o qué es ser un presidente, no había ni goles, ni caballos moviéndose necesariamente en L, ni presidentes. Las reglas constitutivas no describen o regulan acciones: las crean. Y se producen cuando a través del lenguaje las personas llegan a acuerdos que prescriben cómo es que ellos han pensado que debería y les gustaría que se hiciera cierto tipo de cosas en el mundo. Las reglas constitutivas proyectan, a través del lenguaje, el pensamiento humano de que en el mundo se hagan cierto tipo de cosas, cosas que antes no se habían hecho. Así, antes de las reglas constitutivas que expresaban lo que las personas pensaban que debería ser un gol, el movimiento de una ficha del ajedrez, o ser presidente de una república, no había ni goles, ni partidas de ajedrez, ni presidentes de la república. A diferencia de esto, el movimiento de un electrón, o la resistencia de un sólido no se crean cuando el científico natural asume una unidad de medida para caracterizar estas propiedades físicas. Si bien las unidades de medida pueden ser convencionales, lo que describe la unidad de medida: el desplazamiento de los electrones en el cobre o la resistencia de la guadua a la presión del viento, no son convencionales, y han estado en el mundo antes del hombre y su lenguaje. Esto contrasta con el hecho de que las reglas constitutivas no describen sucesos, crean tipos de acciones. Searle al respecto señala: "Las reglas son constitutivas del ajedrez en el sentido de que lo que sea jugar al ajedrez queda en parte constituido por la actuación según esas reglas" (1997:45).

Funciones de estatus. Este es el rasgo decisivo para caracterizar la condición esencial de la región de estudio de las ciencias sociales, y de paso sumar características de la ontología subjetiva. En la función de estatus se funde la Intencionalidad y la capacidad humana de crear Reglas Constitutivas. Las creencias y los deseos, como estados mentales que representan o versan sobre estados de cosas del mundo, así como la capacidad de volcar a través del lenguaje los pensamientos de cómo deben y quieren que se den ciertas cosas en mundo, permiten que las personas impongan a ciertos objetos funciones o capacidades que superan la misma disposición física de esos objetos. Las fibras vegetales y las tintas, por ejemplo, que se aglutinan en los billetes de dinero, en sí mismas no tienen ningún valor, ni mucho menos la función de servir como objeto que media entre los intercambios comerciales. Es solo cuando el hombre se las representa mentalmente como dinero, y cuando colectivamente acepta que a través de ellos se va a ejecutar la acción de acceder a bienes y servicios, que ese pedazo de papel cuenta como dinero. Las fibras vegetales y las tintas aglutinadas como dinero cumplen con una función que no es en ellas natural. No hay nada en la constitución física o química de las fibras vegetales y de las tintas que las determinen naturalmente a que cumplan con la función del intercambio; es una función impuesta por el hombre, y que supera la condición física de los objetos a los que les ha sido impuesta, es una función de estatus: 
"Estas funciones de status se diferencian de las funciones físicas en que un objeto como, por ejemplo, un destornillador, tiene una función física que realiza en virtud de su estructura física, pero las oraciones del castellano, los jaques mates, el dinero o los reyes solo pueden realizar su función si colectivamente se reconoce que tienen determinado status y, con ese status, se les reconoce una función" (Searle 2000:83).

Los Estados, el matrimonio, la economía, las teorías científicas, los partidos políticos, las guerras, las religiones, son hechos en el mundo que en esencia cumplen funciones de estatus, y se dan porque el hombre posee la capacidad de representar los objetos que intervienen en ellos: oraciones o manchas de tinta, humanos, papel, trozos de madera y yeso, de una manera que trasciende la constitución física y química de estos objetos. Es esa trascendencia, esa representación que se dirige a objetos naturales, pero que va más allá de su constitución natural, convirtiéndolos en presidentes, tropas de guerra, proposiciones, crucifijos o figuras para adorar, lo que constituye el repertorio de estudio de las ciencias sociales, y no la constitución física o base material en donde se realizan este tipo de cosas.

La química y la física no son suficientes para crear Estados, matrimonios, teorías científicas, guerras, religiones. Es la capacidad humana de representar y de convenir a través de reglas que ciertos objetos tengan cierto estatus, ciertos poderes de acción, la que constituye la región de estudio de las ciencias sociales. Una región que es ontológicamente subjetiva, pues depende para su existencia de los humanos y sus acuerdos, y que contrasta con la región de estudio de las ciencias naturales, la que es ontológicamente objetiva, pues no depende de las capacidades y los acuerdos humanos para su existencia.

Al inicio de este texto, y siguiendo a Aristóteles, se señaló que los eventos que tienen como causa creencias y deseos (acciones), y que contrastan con los que tienen como origen regularidades naturales (sucesos), marcan diferencias entre las ciencias sociales y las ciencias naturales. Ahora, y siguiendo la posición de Searle, se intentó describir que los hechos que dependen del acuerdo humano, y que revelan una ontología subjetiva, igualmente singularizan la región de estudio de las ciencias sociales. Estados, matrimonio, economía, teorías científicas, partidos políticos, guerras, religiones son ese tipo de hechos. El punto de encaje entre ellos y las acciones, es que este tipo de hechos en esencia son acciones. No son cosas del mundo como los electrones, los átomos, la luna o los océanos. Estados, matrimonio, economía, teorías científicas, partidos políticos, guerras, religiones condicionan ciertas posibilidades para la acción. El Estado es una serie de acciones que organiza social y coercitivamente una serie de instituciones, que a la vez prescriben una serie de acciones que han de ser ejecutadas por los miembros de un territorio. Otro tanto puede decirse del matrimonio, los presidentes, las religiones; trascienden la física y la química de la materia en que se realizan, y se vuelcan al pensamiento como dispositivos que provocan y regulan cierto tipo de comportamientos humanos.

\section{La primera y la tercera persona}

Sumo a la idea de que el objeto de estudio de las ciencias sociales es en esencia ontológicamente subjetivo, en contraste con la ontología objetiva del objeto de estudio de las ciencias naturales, algunas ideas que sugiere el ensayo del filósofo de la mente Thomas Nagel. En su muy comentado trabajo ¿Qué se siente ser murciélago? (2000), Nagel defiende la necesidad de incorporar a la imagen objetiva del mundo la subjetividad de nuestras experiencias, lo que en filosofía se suele asociar como la perspectiva de la primera persona. La idea de asumir la ontología subjetiva como parte ineludible del mundo, como casi todas las cosas en filosofía, no deja de ser polémica, más aún si se lleva hasta el punto de tomarla como condición esencial de los objetos de estudio de las ciencias sociales. En el trabajo citado de Nagel, este se enfrenta a 
las críticas de los reduccionistas y materialistas que consideran el punto de vista de la primera persona como la mala herencia de la filosofía occidental. Nagel se enfrenta a Daniel Dennett, para quien la creencia en la subjetividad renueva las que en su momento fueran las supersticiones medievales, siendo la subjetividad un obstáculo para el conocimiento científico, en especial, para el conocimiento científico del cerebro humano.

En su Libro Dulce sueños Daniel Dennett afirma: “Los métodos de estudio de las ciencias naturales alcanzan para estudiar la conciencia con tanta exhaustividad como cualquier otro fenómeno de la naturaleza, sin dejar residuos significativos. ¿Qué quiere decir aquí "Significativos"? Nada más que esto: si los científicos estudiaran un grano de arena, siempre habrá más cosas que averiguar sobre él, por más tiempo que le dedicaran. En la suma de las fuerzas de atracción y repulsión de todas las partículas subatómicas que componen los átomos del grano, siempre habrá una incertidumbre residual de la magnitud del último dígito significativo que hemos calculado hasta la fecha, y ubicar en tiempo y espacio el grano de arena a través de miles de millones de años lleva a un cono creciente de indiscernibilidad. Sin embargo, esa incertidumbre no será significativa" (2006:43).

El argumento de Nagel contra los que niegan la ontología subjetiva, o la consideran ignorancia residual anodina, es brillantemente simple. Y lo inicia en forma de pregunta: ¿si pudiéramos tener el total de las descripciones relevantes de la constitución y el funcionamiento de la neurofisiología de un murciélago, podría saberse qué se siente ser un murciélago? Públicamente todos tendríamos acceso a la meticulosa descripción científica del sistema nervioso del murciélago, perspectiva de la tercera persona. A pesar de esto, si lo que se quiere es saber qué se siente ser un murciélago, las descripciones públicamente evaluables, dejan aún algo tan real y tan importante de lo que se siente ser murciélago, que negarlo, es tanto como pensar que cuando se lee y se comprenden las descripciones de la neurofisiología de un murciélago se tienen las vivencias del murciélago.

Puede verse la falsa analogía de Dennett del grano de arena con las vivencias de la primera persona, pues lo que él considera ignorancia residual en el grano de arena, es del lado del conocimiento de lo humano lo más significativo: las experiencias subjetivas, las vivencias de las personas, lo que en esencia busca comprender las distintas disciplinas de las ciencias sociales.

Cito a Nagel: "Nuestra propia experiencia nos ofrece el material básico para nuestra imaginación, cuyo campo es, por lo tanto, limitado. No nos ayudaría tratar de imaginar que tenemos membranas en nuestros brazos que nos permiten volar durante el crepúsculo y el amanecer cazando insectos con la boca; que tenemos una vista muy pobre, que percibimos el mundo que nos rodea mediante un sistema de señales sonoras de alta frecuencia, y que nos pasamos el día en un desván colgados boca abajo sujetos de los pies. En la medida en que puedo imaginarme todo esto, que no es mucho, me indica tan sólo cómo sería para mí comportarme como un murciélago. Pero esa no es la cuestión. Deseo saber qué se siente para un murciélago ser un murciélago" (2000:280).

Puede desestimarse por trivial la idea de Nagel de que sentirse humano no es sentirse murciélago. Ser triángulo no es ser círculo. Y claro, como Nagel y cualquier otro lector de su ensayo es humano, tendrá que concluir como él, que nunca se podrá saber qué se siente ser murciélago. Una idea que suena a perogrullada. Pero el punto no es si podemos o no podemos saber qué se siente ser murciélago, sino que además del conocimiento público que se puede tener del murciélago, el conocimiento que tiene como clase referencia la neurofisiología del murciélago, existe otro tipo de clase referencia que no es subsumible 
en la teoría neurofisiológica, o en las descripciones en tercera persona que la ciencia natural puede hacer. La experiencia consciente, la subjetividad de la experiencia, la que se percibe y se vive en primera persona, y que a través del lenguaje intenta hacerse pública, pero que no obstante, y como lo señala Nagel, no queda plenamente subsumida en él, es parte de la ontología subjetiva que constituye la ontología de la realidad social. Y es tan real como las montañas y rocas.

\section{Lenguaje y ontología de las ciencias sociales}

En Palabras y acciones, John Austin (1971) hizo una de las críticas demoledoras a la tesis de algunos positivistas lógicos de que el único lenguaje con sentido es aquel conformado por enunciados verificables. Denominó Falacia descriptiva, al intento argumentativo de asumir que los enunciados que no describen estados de cosas del mundo son pseudo enunciados, y en el mejor de los casos, absurdos que tienen como propósito despertar emociones. Austin mostró que con el lenguaje no sólo se describe el mundo, sino que también hacemos cosas en el mundo. Y si bien, en algunos casos el lenguaje se usa para describir hechos del mundo, en otros casos el lenguaje es usado con otros propósitos, como prevenir sobre la ocurrencia de ciertos sucesos. Es falacioso considerar que como el uso descriptivo del lenguaje ha sido promisorio en la teorización científica del mundo, lo que no cumpla esta función lingüística carece de sentido no haciendo parte de lo que genuinamente puede ser entendido como lenguaje. Esto es confundir la parte con el todo. Las descripciones son sólo una, entre la gran gama de las funciones del lenguaje. Austin denominó expresiones realizativas a todas aquellas oraciones que pronunciadas o escritas tienen la función no de describir o decir algo del mundo, sino de crear o hacer ciertas cosas en el mundo.

Propongo denominarla oración realizativa o expresión realizativa o, para abreviar, "un realizativo". La palabra "realizativo" será usada en muchas formas y construcciones conectadas entre sí, tal como ocurre con el término "imperativo". Deriva, por supuesto, de "realizar", que es el verbo usual que se antepone al sustantivo "acción". Indica que emitir la expresión es realizar la acción y que ésta no se concibe normalmente como el mero decir algo (Austin 1971:47).

Uno de los ejemplos favoritos de Austin es el de bautizar un barco. Cuando la persona indicada y en el momento indicado emite la expresión "Bautizo este barco Queen Elizabeth" no está haciendo la descripción de un vehículo flotante, está bautizando un barco. En este caso las palabras no dicen cosas, hacen cosas: Bautizar. La teoría de Austin, que busca en un inicio poner en su justo valor el criterio de sentido que adoptaron positivistas como Carnap en su influyente ensayo La superación de la metafísica mediante el análisis lógico del lenguaje o Alfred Ayer en La eliminación de la metafísica, hizo ver en el lenguaje no solo un instrumento de descripción, sino también de creación. El lenguaje crea cosas.

Al afirmar que con el lenguaje se hacen cosas, que con él se constituyen modos de ser en el mundo, saltan a la vista las implicaciones ontológicas. Es John Searle el que abraza las implicaciones ontológicas de las tesis de Austin. El paso de Searle del lenguaje a la ontología es brillantemente simple. Si el lenguaje cumple, entre otras, la función de hacer cosas con palabras, entonces, además de los hechos que han existido con independencia de los humanos, existen cosas que en tanto hechas con lenguaje dependen de él y de los humanos en tanto su usuario.

En Actos de habla John Searle inicia el desarrollo de esta idea sugerida en el análisis de Austin sobre las expresiones realizativas, idea que alcanza su plena formulación filosófica en el libro ya clásico sobre filosofía de la sociedad: La construcción de la realidad social. En el contexto que nos interesa, el de las 
ciencias sociales y su contraste con las ciencias naturales, lo que puede verse intuitivamente es que la clase referencia de las teorías de las ciencias sociales son hechos dependientes del lenguaje, intuición que sumada a lo dicho arriba sobre lo ontológicamente subjetivo de los objetos de estudio de las ciencias sociales, aclara aún más la distinción ontológica entre ciencias sociales y ciencias naturales.

Los objetos de estudio de las ciencias sociales, hechos institucionales o acciones, como he querido mostrar arriba, son ontológicamente subjetivos, pues una condición esencial de su existencia es el pensamiento humano. Son objetos que en esencia están hechos de pensamiento. Esto contrasta con el objeto de estudio de las ciencias naturales, en donde el pensamiento es irrelevante para su existencia.

Se hace más visible esta diferencia si sumamos la idea que esas porciones del pensamiento que constituye los objetos de estudio de las ciencias sociales, a su vez, dependen del lenguaje. Así, las cosas que estudian las ciencias sociales, a diferencia de las cosas que estudian las ciencias naturales, dependen del lenguaje. Los Estados, el matrimonio, la economía, las teorías científicas, los partidos políticos, las guerras, las religiones, son cosas que están hechas con lenguaje. Los electrones, los átomos, la luna o los océanos, existen sin lenguaje.

La manera de justificar esta nueva distinción también la podemos encontrar en la propuesta del filósofo John Searle. Su idea es que si bien hay pensamientos que existen con independencia del lenguaje, como el dolor, la ansiedad, hay pensamientos que dependen esencialmente del lenguaje: "Sofía es una palabra de 5 letras", es un pensamiento que depende del lenguaje. Sólo puede tenerse este pensamiento si existe la palabra Sofía. Mientras que puede existir el dolor o la ansiedad con independencia de cualquier palabra.

Esas porciones del pensamiento que trascienden la constitución física y química de las cosas, constituyendo, por ejemplo, el dinero, son justamente porciones del pensamiento que dependen del lenguaje y que hacen que esos hechos dependan del lenguaje. Todo porque la función de estatus y las reglas constitutivas que tienen como base los estados intencionales, sólo pueden realizarse a través del lenguaje. Un billete de 50 mil pesos es el trozo de papel más el pensamiento colectivo de las personas de que ese trozo de papel cuenta o vale como 50 mil pesos, y a su vez, ese pensamiento solo puede darse en tanto las personas cuenten en su mente con los símbolos pesos y 50 mil. Para las personas representar en la masa corporal de Juan Manuel Santos al presidente de Colombia, y que asuman sus mandatos como disposiciones para la acción, es necesario que compartan una serie de pensamientos comunes, que sólo pueden darse y realizarse a través del lenguaje. La propiedad privada, las religiones, los Estados, los presidentes, son en esencia las representaciones comunes que las personas a través del lenguaje se hacen de ciertos hechos físicos y químicos del mundo. Representaciones que se instauran en la mente de las personas como nuevos poderes causales para cierto tipo de acciones y que sólo pueden ser colectivos a través del lenguaje.

Los objetos de estudio de las ciencias sociales están en esencia constituidos por porciones de pensamientos, pensamientos que a su vez dependen del lenguaje. Pues sólo a través de él se puede dar el reconocimiento público de los pensamientos propios y de otros. Pensamientos que en conjunción permiten otorgar a ciertos objetos materiales un estatus que trasciende las propiedades físicas y químicas de ellos, convirtiéndolos en hechos institucionales o acciones, los que dadas sus características son los objetos de estudio de las ciencias sociales. 
Dos son, entonces, las características que desde la filosofía, y específicamente desde lo que se ha aclarado como ontología, pueden extraerse de las ciencias sociales que las hacen inconmensurables con las ciencias naturales. La primera es que los objetos de estudio de las ciencias sociales son ontológicamente subjetivos, esto es, su modo de existencia es dependiente de las personas, mientras que los objetos de estudio de las ciencias naturales son ontológicamente objetivos, su modo de existencia es independiente de los sujetos. La ontológica subjetiva se da porque solo a través de ciertos dispositivos mentales la realidad social puede existir. Y la segunda característica es que los hechos que son estudiados por las ciencias sociales dependen del lenguaje, sólo pueden existir en tanto haya un sistema de símbolos que haga públicos los pensamientos de las personas, en contraste con los hechos y eventos que estudian las ciencias naturales, los cuales no necesitan del lenguaje para existir.

\section{Nota}

Este texto es parte de los resultados del proyecto de investigación "Ontología de la realidad social, una perspectiva de investigación para las ciencias sociales", aprobado y financiado por la vicerrectoría de investigaciones de la Universidad del Quindío.

\section{Bibliografía}

Aristóteles (1967) Obras completas. Madrid: Aguilar.

Austin, J. (1971) Palabras y acciones. Buenos Aires: Paidós.

Dennett, D. 2006. Dulce sueños, obstáculos filosóficos para una ciencia de la conciencia. Buenos Aires: Katz.

Frankfurt, H. 2006. La importancia de lo que nos preocupa. Buenos Aires: Katz.

Nagel, T. 2000. Ensayos sobre la vida humana. México: Fondo de Cultura Económica.

Searle, J. (1992) Intencionalidad, un ensayo en la filosofía de la mente. Madrid: Tecnos.

Searle, J. (1997) La construcción de la realidad social. Barcelona: Paidós.

Searle, J. 2000. Razones para actuar: Una teoría del libre albedrío. Oviedo: Ediciones Nobel.

Recibido el 13 Mar 2014

Aceptado el $25 \mathrm{Jul} 2014$ 